\title{
Association of small intestinal diverticulosis with chronic pancreatitis leading to severe malabsorption. Report of three cases.
}

\author{
Y.R. Mahida, R.W. Chapman and D.P. Jewell \\ Gastroenterology Unit, Radcliffe Infirmary, Oxford OX2 6HE, UK.
}

\begin{abstract}
Summary: Three cases of chronic pancreatitis occurring in patients with small intestinal diverticulosis and bacterial overgrowth are reported. In two of the cases, pancreatic supplements were therapeutically beneficial (the third being unable to tolerate them). Two of the patients also developed diverticular perforation. The possible nature of the association between small intestinal diverticulosis and chronic pancreatitis is discussed.
\end{abstract}

\section{Introduction}

Jejunal diverticulosis is uncommon, with an autopsy incidence of approximately $0.5 \% .^{1}$ Although the diverticula do not usually cause symptoms, multiple large diverticula may lead to bacterial overgrowth ('blind loop') and malabsorption particularly of fat and vitamin $B_{12} \cdot{ }^{1}$

We report three cases of severe malabsorption apparently caused by bacterial overgrowth occurring in patients with jejunal diverticulosis who, in addition, were found to have chronic pancreatitis.

\section{Case reports}

Case 1

A 78 year old Caucasian woman developed steatorrhoea and macrocytic anaemia secondary to vitamin $B_{12}$ deficiency in 1968. A diagnosis of bacterial overgrowth secondary to jejunal diverticulosis was made on the basis of a small bowel follow through examination. She was treated with antibiotics, vitamin $B_{12}$ injections and dietary supplements with good effect. Subsequently she had frequent episodes of steatorrhoea which were treated in the same manner. In 1971, she presented with acute abdominal pain and was found to have a perforated jejunal diverticulum which was closed at laparotomy.

In 1981, in view of persistent malabsorption despite treatment with antibiotics, pancreatic function was assessed by means of a Lundh test meal.

Correspondence: D.P. Jewell, D.Phil., F.R.C.P. Accepted: 19 May 1988.
The results showed pancreatic insufficiency and pancreatic enzyme replacement with Nutrizym was started.

In 1986, she was referred to the John Radcliffe Hospital complaining of anorexia, weight loss, and intermittent abdominal pain. Whilst taking a low fat diet she had one bowel motion a day, but on a normal diet, she complained of steatorrhoea. She denied any alcohol intake.

On examination, she was very thin with pitting ankle oedema. Abdominal examination revealed visible peristalsis. Investigations revealed a normal haemoglobin, and white blood count, erythrocyte sedimentation rate (ESR) was $26 \mathrm{~mm} / \mathrm{h}$. Random blood sugar was elevated at $16.4 \mathrm{mmol} / 1$ and remained so. Urea and electrolytes were normal. Serum albumin was $34 \mathrm{~g} / 1$ (normal $35-50 \mathrm{~g} / \mathrm{l}$ ), the other liver function tests were normal. Serum $B_{12}$, folate and iron level were normal as she was already on $\mathbf{B}_{12}$ injections and iron supplements. Ultrasound scan of the upper abdomen was normal. A small bowel enema showed gross jejunal diverticulosis (Figure 1). Culture of a duodenal aspirate grew a significant mixed growth of coliforms and faecal streptococci. A jejunal biopsy specimen was normal.

Endoscopic retrograde cholangiography (ERCP) revealed multiple diverticula in the second part of the duodenum with two large diverticula on either side of the ampulla of Vater. The main pancreatic duct and the side branches were dilated and irregular, being diagnostic of chronic pancreatitis (Figure 2 ). The biliary system was normal, no stones were seen in the gall bladder.

(C) The Fellowship of Postgraduate Medicine, 1988 


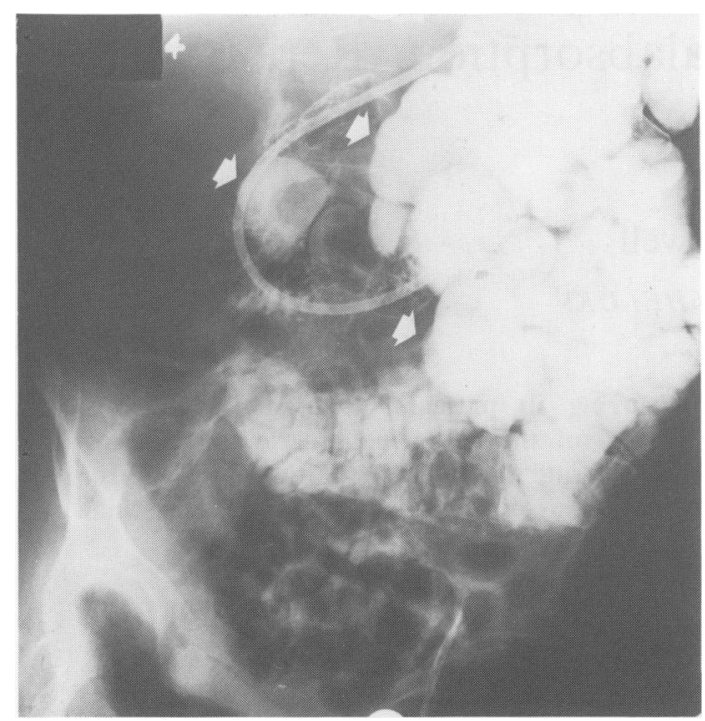

Figure 1 Small bowel enema of case 1 showing large jejunal diverticula (arrowed).

Twenty-four hour stool weights (mean of 3 days collection), on a low fat diet, without and with pancreatic supplements were $513 \mathrm{~g}$ and $295 \mathrm{~g}$ respectively indicating a significant pancreatic component to her fat malabsorption.

She was treated with metronidazole, pancreatic supplements (Nutrizym) and a high calorie, high protein, low fat diet. Her diabetes mellitus was controlled with tolbutamide. She has since had one episode of symptomatic steatorrhoea requiring a repeat course of metronidazole.

\section{Case 2}

A 63 year old Caucasian man had suffered recurrent bouts of abdominal pain and distension, flatulence and diarrhoea for about 12 years. In 1980, jejunal diverticulosis was diagnosed on the basis of a small bowel follow through examination. He was treated with tetracycline with good effect. A course of metronidazole and ampicillin was required in 1984 for a recurrence of symptoms.

In 1986, he was referred to the John Radcliffe Hospital complaining of central abdominal pain and distension, nausea, flatulence, and weight loss of $6 \mathrm{~kg}$ over the previous 12 months. On direct questioning, he admitted to intermittent loose or liquid bowel motions which were occasionally pale. He drank alcohol only once or twice a year. On examination, he was very thin with a distended abdomen due to ascites.

Investigations revealed a normal haemoglobin, ESR, serum urea and electrolytes, liver functiom tests and serum albumin. Serum levels of vitamio $\mathrm{B}_{12}$, folate iron and iron binding capacity were alsor. all normal. Prothrombin time was also normal. NoD excess fat globules were detected in a specimen of stool. Abdominal X-ray showed fluid levels as well as widespread intraperitoneal gas. Chest X-ray

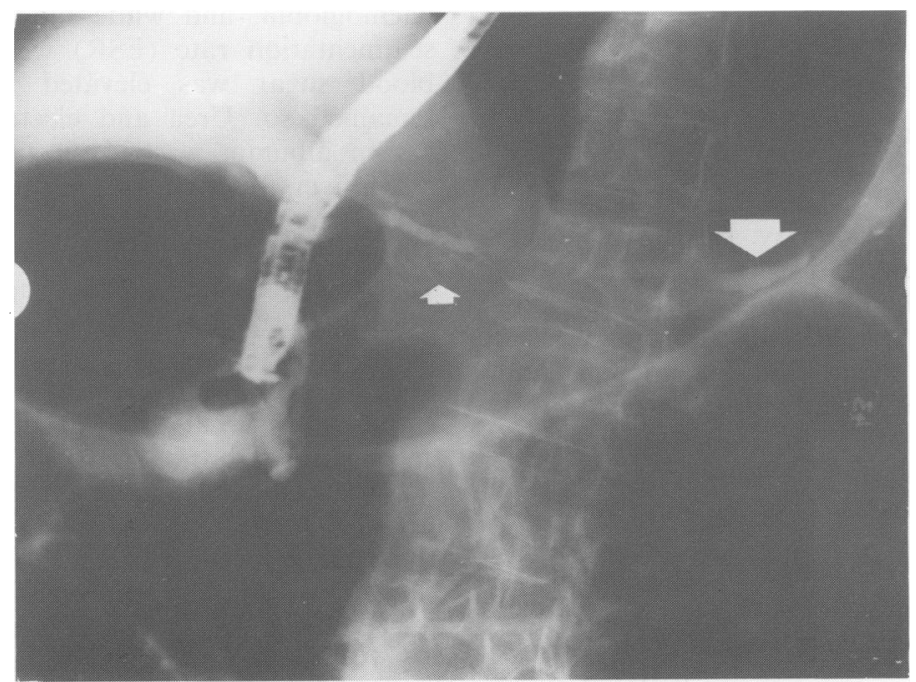

Figure 2 Pancreatogram of case 1 showing dilatation of main pancreatic duct (large arrow) and irregular side branches (small arrow). 
revealed air under the diaphragm. Ultrasound examination confirmed the presence of ascites but no other abnormality was found. Ascitic tap revealed a protein level of $47 \mathrm{~g} / 1$ with a normal amylase level and culture of the fluid revealed no growth. Endoscopic biopsy of the second part of the duodenum was normal. Culture of a duodenal aspirate grew a significant growth of two types of coliforms.

ERCP showed a normal ampulla of Vater with a large periampullary diverticulum. The biliary tree was normal with no stones in the gallbladder. However, the main pancreatic duct was irregular with strictured and dilated side branches, diagnostic of chronic pancreatitis.

He was treated with cephradine, pancreatic supplements (Pancrex V forte), ranitidine and a high calorie, high protein, low fat diet. The ascites and intraperitoneal gas were thought to be due to a perforated jejunal diverticulum and they resolved spontaneously over a few days.

One month after discharge, he was admitted as an emergency with abdominal pain to another hospital where he underwent a laparotomy. This revealed infarcted gut secondary to volvulus of the small intestine. Three feet of small bowel and ascending colon were resected. He made a good post-operative recovery, and remains well.

\section{Case 3}

A 74 year old Caucasian man was admitted as an emergency to the John Radcliffe Hospital in 1983. He gave a 2-year history of frequent loose bowel motions and a one week history of profuse liquid diarrhoea. Alcohol was consumed only occasionally ( 2 to 3 times a year). On examination, he was clinically anaemic, thin, with a liver edge which was palpable $4 \mathrm{~cm}$ below the right costal margin. Sigmoidoscopy showed pale stools with normal rectal mucosa.

Investigations revealed a haemoglobin of $10 \mathrm{~g} / \mathrm{dl}$ (normal 13.3-17.7 g/dl) with an elevated mean corpuscular volume (MCV) of $132 \mathrm{fl}$ (normal 80.5$99.7 \mathrm{fl}$ ). Serum albumin was $25 \mathrm{~g} / 1$ (normal $35-50$ $\mathrm{g} / \mathrm{l})$, but other liver function tests were normal. Examination of a stool specimen revealed excess fat globules. Vitamin $\mathrm{B}_{12}$ studies using a total body counter showed reduced absorption with no response to intrinsic factor. Serum iron level was $12.5 \mu \mathrm{mol} / 1$ (normal range $14-31 \mu \mathrm{mol} / \mathrm{l}$ ), total iron binding capacity was $30.0 \mu \mathrm{mol} / 1$ (normal range 54 $75 \mu \mathrm{mol} / \mathrm{l})$. Small bowel enema showed several jejunal diverticula (Figure 3). A lactulose breath test was performed. This showed an early peak of breath hydrogen at 30 minutes, compatible with bacterial contamination of the small bowel.
He was treated with metronidazole and vitamin $B_{12}$ injections and a high calorie, low fat diet. However, he required codeine phosphate to control the diarrhoea and stool examination continued to show excess fat globules. An ERCP was therefore performed. The ampulla of Vater was hidden under a prominent fold but no periampullary diverticula were seen. The pancreatic duct was dilated with abnormal side branches. The biliary tree and gall bladder were normal (Figure 4).

He was started on pancreatic supplements but was unable to tolerate them, as he developed severe

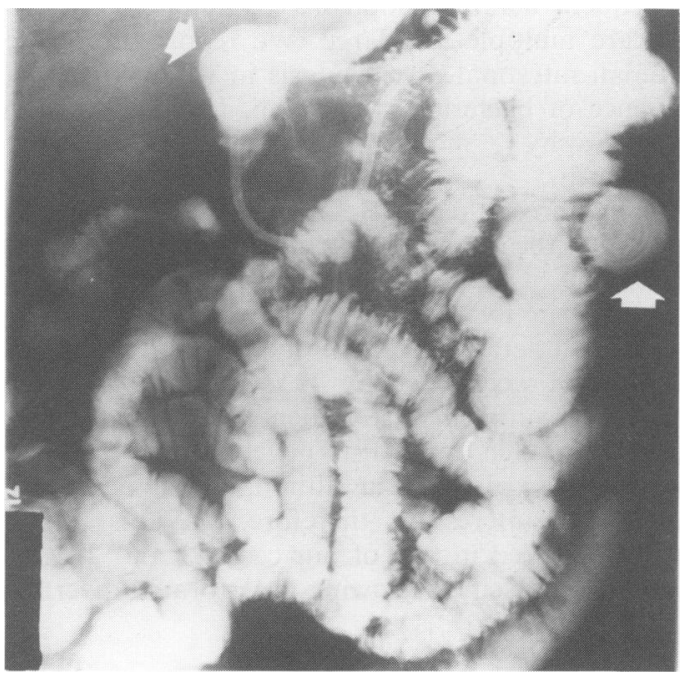

Figure 3 Small bowel enema of case 3. Arrows point to two large diverticula.

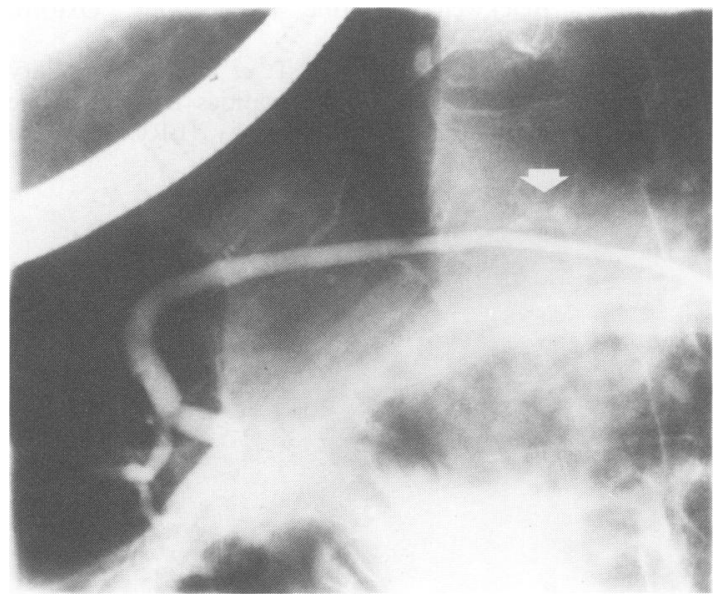

Figure 4 Pancreatogram of case 3. Arrow points to irregular and dilated side branches. 
mouth ulceration. Soon afterwards, he sustained a fractured neck of femur and died post-operatively.

At post-mortem, the cause of death was pulmonary embolism. The diagnosis of chronic calcific pancreatitis was confirmed and the presence of jejunal diverticula noted.

\section{Discussion}

Small intestinal diverticula are usually acquired and often occur beyond the fifth decade. ${ }^{1}$ They are often asymptomatic but bacterial overgrowth and subsequent malabsorption may occur if the diverticula are multiple and large. We report three cases of small intestinal diverticulosis in whom there was evidence of bacterial overgrowth. In addition, pancreatography showed changes of chronic pancreatitis.

In all three, there were no other factors predisposing to the development of chronic pancreatitis. Pancreatic supplements were therapeutically beneficial in two of the patients (the third being unable to tolerate them). In one patient (case 1) the mean stool weight fell by $40 \%$ to $295 \mathrm{~g}$ a day on pancreatic supplements. Patients with small intestinal diverticula may also develop perforation of one of these diverticula although this is uncommon. ${ }^{2}$ It is interesting, therefore, that this complication occurred in two of our cases (1 and 2). Case 2 developed ascites following a perforated diverticu-

\section{References}

1. Tabaqchali, S. \& Booth, C.C. Bacterial overgrowth. In: Booth, C.C. and Neale, G. (eds) Disorders of the Small Intestine. Blackwell Scientific Publications, Oxford, 1985, pp 249-270.

2. Osnes, M., Myren, J., Lotveit, T. et al. Juxtapapillary duodenal diverticula and abnormalities by endoscopic retrograde cholangio-pancreatography (ERCP). Scand J Gastroenterol 1977, 12: 347-351. lum and the ascitic fluid and intraperitoneal gas resolved spontaneously over a few days. A few weeks later, he also developed another complication of this condition, volvulus of the small intestine, requiring surgical correction.

Over the last few years, several studies have demonstrated a relationship between juxtapapillary diverticula and gallstone disease. ${ }^{2-4}$ Two of our cases had duodenal diverticula closely associated with the ampulla of Vater but there was no evidence of gallstone disease in either.

The nature of the association between small intestinal diverticula and chronic pancreatitis is unknown. However, it is interesting to speculate that longstanding bacterial overgrowth with reflux into the pancreatic duct may predispose to chronic pancreatitis. This is supported by the fact that in the first two cases, with documented longstanding diverticula and bacterial overgrowth, a further deterioration led to the diagnosis of chronic pancreatitis. Another possible explanation could be that the periampullary diverticula present in two of the cases could have impaired drainage at the ampulla and may have led to an obstructive pancreatopathy.

In any event it should be borne in mind that chronic pancreatitis in patients with small intestinab diverticulosis is another potential cause of malo absorption. It should be actively excluded in patients with bacterial overgrowth due to smale intestinal diverticulosis who are not responding to antibiotic treatment.

3. van-der-Spuy, S. The relationship between juxtapapillary diverticula and biliary calculi. An endoscopic study. Endoscopy 1979, 11: 197-202.

4. Osnes, M., Lotveit, T., Larsen, S. et al. Duodenal diverticula and their relationship to age, sex and biliary calculi. Scand J. Gastroenterol 1981, 16: 103-107. 